Original article

\title{
MORPHOLOGICAL STUDY OF THE MIDBRAIN TECTUM IN OSTRICH (STRUTHIO CAMELUS) EMBRYO
}

\author{
Z. KOUSHAFAR ${ }^{1} \&$ A. A. MOHAMMADPOUR ${ }^{2}$ \\ ${ }^{1} \mathrm{PhD}$ Student of Histology, ${ }^{2}$ Department of Basic Science; Faculty of \\ Veterinary Medicine, Ferdowsi University of Mashhad, Mashhad, Iran
}

\section{Summary}

Koushafar, Z. \& A. A. Mohammadpour, 2019. Morphological study of the midbrain tectum in ostrich (Struthio camelus) embryo. Bulg. J. Vet. Med., 22, No 2, 143-151.

In this study the morphological features of the optic tectum in ostrich embryo were studied macroscopically and microscopically. After gross anatomical study, fixed specimens of the optic lobes in $30^{\text {th }}, 36^{\text {th }}$ and $40^{\text {th }}$ embryonic days were processed for paraffin sections. Sections were stained by Harris haematoxylin and eosin (H\&E), Luxol Fast Blue/Cresyl Echt Violet and Malory PTAH dyes. The optic lobes had large volumes even on the $30^{\text {th }}$ embryonic day and increased proportionally to age. The optic lobe consisted of two parts: gray matter (outer) and white matter (inner). The first external layer of the optic lobe e.g. molecular layer consisted of neural fibres, neuroglia and scarce small neurons. The most common appearance of the optic lobes was characterised by small to medium-sized neurons (rounded to pyramid-shaped with large and pale nucleus consistong of obvious nucleoli arranged in three layers whose thickness increased in the deeper one) supported by neuroglia. Larger size neurons and occasionally multipolar neurons were presented in the interior compared with these layers. The lateral mesencephalic nucleus was detectable in the optic lobe base even on $30^{\text {th }}$ embryonic day and was composed of few multipolar neurons supported by neuroglia. The tectal ventricles were lined with simple cuboidal ciliated ependymal cells in the embryonic period. As embryonic age increased, the ratio of tectal ventricle volume to its thickness decreased. Special stainings showed that Nissl bodies and myelin fibres, also glial fibres were available from the $30^{\text {th }}$ embryonic day and that their density, especially myelin fibres density, increased with age.

Key words: embryo, morphology, optic lobe, ostrich, tectum

\section{INTRODUCTION}

The ostrich (Struthio camelus) is the largest living bird in the world raised primarily for meat, besides, its hide (leather) and fat (oil) are considered as byproducts. The North American ostrich industry is relatively young and locally produced ostrich products have only been in the markets since the early 1980s. In the last two decades, people have begun to pay more attention to ostriches as a result of their great economic value. The ostrich breeding industry is growing rapidly in many places, including the USA, Australia, New Zealand, Canada, Europe, and 
China (AlNasser et al., 2003; Bejaei \& Cheng, 2014). Birds have a three-part brain composed of segments for olfaction, vision and hearing. The relative proportions of each of these sections vary with the ecology of the bird. For example, vultures and falcons, who detect low levels of methane gas, have a large olfactory section to their brains while some birds like ostriches need to have a very well developed sense of vision for living in deserts (Martin et al., 2007; Douglas et al., 2009). The roof of the midbrain or tectum, develops as the primary visual center. The optic lobes, especially prominent in fish and birds, are a part of this area. In fish and amphibians the tectum is the major centre of the nervous system and offers the greatest influence on body activity. While this area is still significant in reptiles and birds, it is supplanted in importance by the cerebral hemispheres (Hall, 2008). As a result of its importance in many aspects of visual processing, there have been numerous anatomical, immunohistochemical, developmental and electrophysiological studies of the avian tectum (Letelier et al., 2000; Sebesteny et al., 2002; Luksch, 2003; Metzger et al., 2006; Wang et al., 2006; Khanbabaie et al., 2007; Manns et al., 2007; Douglas et al., 2009). Ostriches have large-sized eyes with high visual ability. There are some reports on the external features of the African ostrich brain (Wenqin et al., 2005; Peng et al., 2010) and the internal features and the histological structures of the brain parts (Karakoura et al., 2015). However, little information is available regarding central nervous system of the ostrich, neither there are histomorphological studies on the optic tectum, especially during embryonic period. Therefore, the aim of this study is to make a spotlight on the gross anatomy of the optic tecta, their histologi- cal structures, and existence of Nissl bodies, myelin fibres, and glial fibres in these parts of the brain of ostrich during the embryonic period.

\section{MATERIALS AND METHODS}

Fifteen eggs in $30^{\text {th }}, 36^{\text {th }}$, and $40^{\text {th }}$ embryonic days of the Canadian ostrich were obtained from Toos farm in Mashhad, Iran (five eggs in each age). Eggs were windowed and embryos carefully cut free from the yolk and then placed in a culture dish containing Ringer's solution. Extraembryonic membranes were dissected away and embryos rinsed to remove remaining yolk in Ringer's solution. The cranial cavity was opened very carefully with the help of forceps, scissors, and scalpel. The meninges covering the brain and their attachments with cranial bones were cut. The brain samples were cleaned (washed) in normal saline solution, then the gross anatomical study was performed. For histological examination, samples of optic lobes were fixed in $10 \%$ phosphate-buffered formaldehyde. The fixed specimens were processed for paraffin sections. Tissue processing machine performed three main tissue processing steps, namely: dehydration, clearing and infiltration. Dehydration was carried out by immersing tissue in a series of ethanol solutions of increasing concentrations until water-free $(100 \%)$ alcohol was reached. Following the dehydration, the tissue was immersed in one to three different xylene immersions for clearing. Infiltration was when the final xylene was replaced with melted wax infiltrating the tissue. After infiltration, the tissue was transferred to an embedding station for blocking.

Five micrometer thick sections were prepared and stained by Harris haema- 
toxylin and eosin (H\&E), Luxol Fast Blue/Cresyl Echt Violet and Malory phosphotungstic acid haematoxylin (PTAH) as per standard methoda (Bancroft et al.,1996). Luxol Fast Blue/Cresyl Echt Violet staining was done for demonstration of both myelin and Nissl substances in tissue sections. On stained sections, myelin sheath was stained blue and Nissl bodies and nuclei: violet. Malory PTAH staining demonstrated glial fibres in tissue sections (blue), nuclein (bleu), neurons (salmon) and myelin (blue).

Photography was carried out from the selected fields both under lower $(\times 40)$ and higher $(\times 1000)$ magnifications under Leica DM 2500 microscope (Germany)

\section{RESULTS}

\section{Macroscopical results}

In ostrich embryo, the optic lobes were developed at all studied embryonic ages and incompletely buried under cerebral hemispheres. The optic lobes were large oval structures laterally. These structures were created from the midbrain drawn-out dorsally between the caudal poles of cerebral hemispheres rostrally and cerebellum caudomedially. Dorsal transverse fissure separated optic lobes from cerebellum and cerebral hemispheres (Fig. 1-3). The volume of the optic tectum increased parallelly to age.

\section{Microscopical results}

The optic tectum consisted of two parts: gray matter and white matter, the gray matter was situated on the outer while the white matter - on the inner part (Fig. 9). The external layer was molecular layer (Figs 4, $7 \&$ \&) and the most common appearance of the cortex of optic lobe was characterised by small to medium-sized

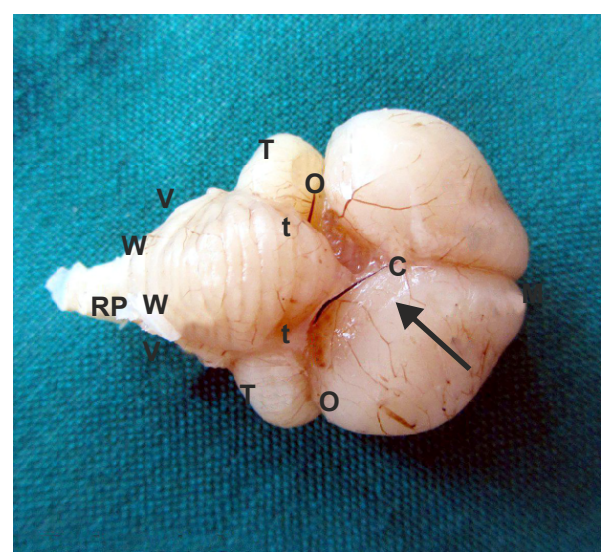

Fig. 1. Dorsal view of the ostrich brain, $30^{\text {th }}$ embryonic day. T: cerebral hemisphere, C: cerebellum, M: medulla oblongata,O: optic lobe, RP: rostral cerebral pole, t: Dorsal transverse fissure, V: vallecula, W: Wulst, arrow: cerebellar folia.

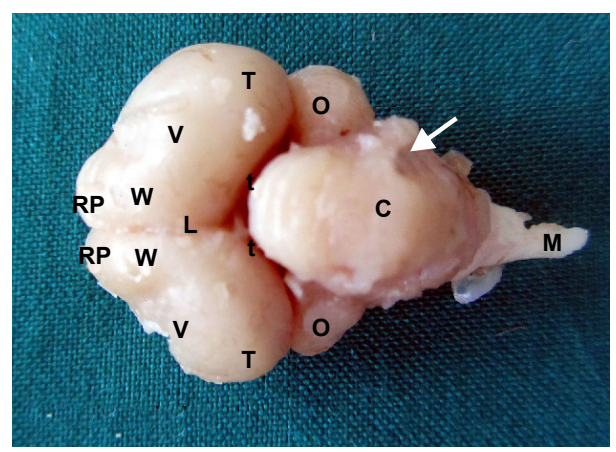

Fig. 2. Dorsal view of the ostrich brain, $36^{\text {th }}$ embrionic day: cerebral hemisphere, C: cerebellum, M: medulla oblongata, O: optic lobe, RP: rostral cerebral pole, L: dorsal cerebral longitudinal fissure, t: Dorsal transverse fissure, V: vallecula, W: Wulst, arrow: cerebellar folia.

neurons (with a central nucleus and obvious nucleolus) supported by neuroglia (Fig. 5). The size of neurons was estimated by comparison to the size of glial 
cells. The neurons were mostly rounded and at a lesser extent - pyramidally shaped. The optic lobe cortex showed a special arrangement for neurons: small to medium-sized neurons were arranged in three layers and the thickness of these layers increased toward the deeper layers

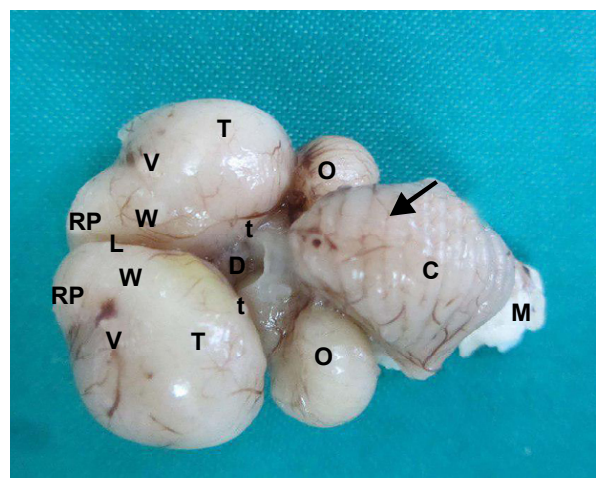

Fig. 3. Dorsal view of the ostrich brain, $40^{\text {th }}$ embrionic day. T: cerebral hemisphere, C: cerebellum, M: medulla oblongata, O: optic lobe, RP: rostral cerebral pole, L: dorsal cerebral longitudinal fissure, $\mathrm{t}$ : dorsal transverse fissure, $\mathrm{V}$ : vallecula, W: Wulst, MB: interlobar midbrain, D: diencephalon, arrow: cerebellar folia.

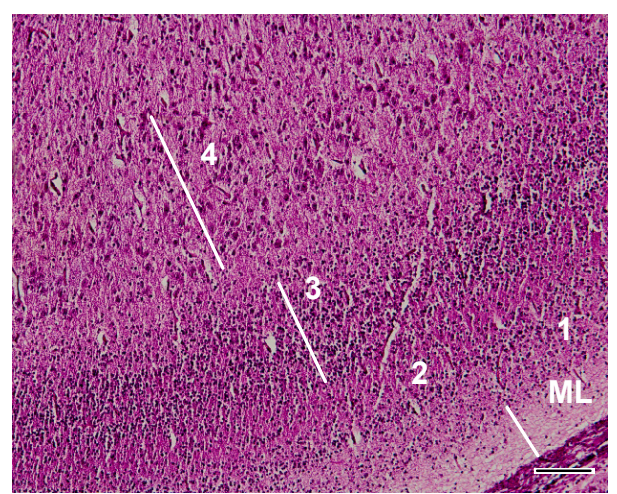

Fig. 4. Optic lobe $\left(30^{\text {th }}\right.$ embryonic day) was formed from small to medium-sized neurons with few neuroglia arranged in three layers (1, $2,3)$. There are larger sized neurons in the inner layer (4). ML: Molecular layer (H\&E, bar $=25 \mu \mathrm{m})$.

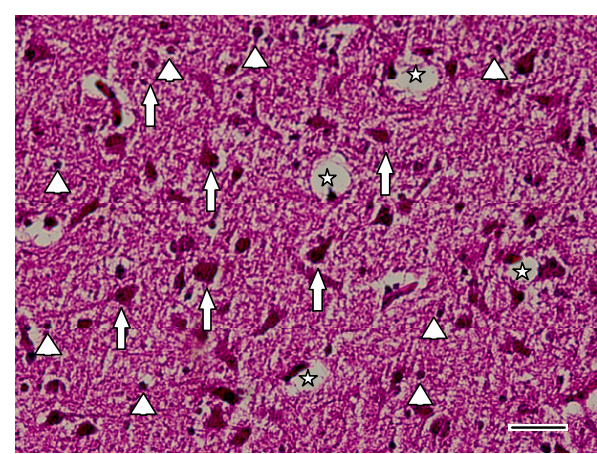

Fig. 5. Optic lobe ( $30^{\text {th }}$ embryonic day) was formed from medium-sized pyramidal neurons with few neuroglia. Arrows: neuron; arrowheads: neuroglia; stars: prevascular space $(\mathrm{H} \& \mathrm{E}, \mathrm{bar}=5 \mu \mathrm{m})$.

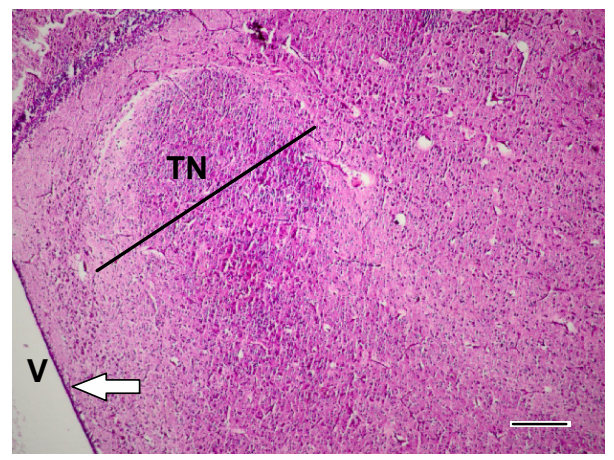

Fig. 6. The tectal nucleous $(\mathrm{TN})$ at the base of the optic lobe, $30^{\text {th }}$ embryonic day. V: tectal ventricle; arrow: ependymal layer (H\&E, bar $=50 \mu \mathrm{m})$.

(Figs $4,7 \& 8$ ). Following these layers, larger neurons were presented internally and occasionally, few multipolar neurons were found in this part (Figs 4, 7-10). Prevascular spaces were detectable from the $30^{\text {th }}$ embryonic day (Fig. 5). The large-sized nucleus (lateral mesencephalic nucleus) was visible in the base of optic lobe even on the $30^{\text {th }}$ embryonic day (Fig. 6). This tectal nucleus was composed of few multipolar neurons supported by neuroglia (Fig. 9). The tectal ventricles were covered with simple cuboidal ciliated 
ependymal cells in three ages in our study (Figs $6 \& 10$ ). As embryonic age increased, the optic lobe thickness increased too and the ratio of tectal ventricle volume to its thickness decreased.

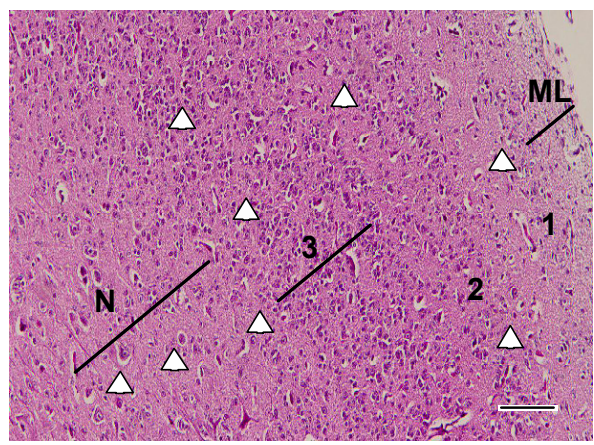

Fig. 7. Optic lobe $\left(36^{\text {th }}\right.$ embryonic day) was formed from small to medium-sized neurons with few neuroglia arranged in three layers $(1$, $2,3)$. There are larger sized neurons in the inner layer $(\mathrm{N})$. ML: molecular layer $(\mathrm{H} \& \mathrm{E}$, bar $=25 \mu \mathrm{m})$.

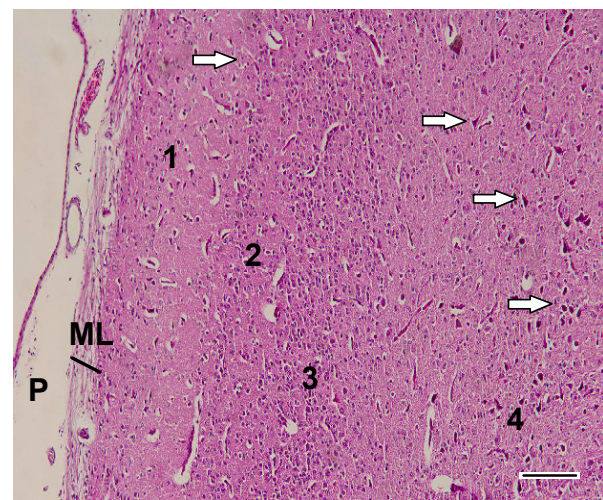

Fig. 8. Optic lobe $\left(40^{\text {th }}\right.$ embryonic day) was formed from medium sized neurons with few neuroglia arranged in three layers $(1,2,3)$. There are larger sized and occasionally multipolar neurons (arrow) in the inner layer (4). ML: molecular layer, P: pia matter $(\mathrm{H} \& \mathrm{E}$, bar $=25 \mu \mathrm{m})$.

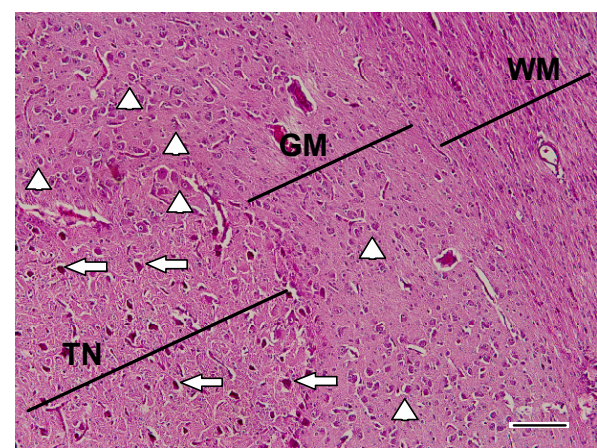

Fig. 9. Optic lobe (40 ${ }^{\text {th }}$ embryonic day) contains large-size neurons (head arrows) in the inner layer. The tectal nucleus (TN) was composed of few multipolar neurons (arrows) supported by neuroglia. GM: gray matter,WM: white matter $(\mathrm{H} \& \mathrm{E}, \mathrm{bar}=20 \mu \mathrm{m})$.

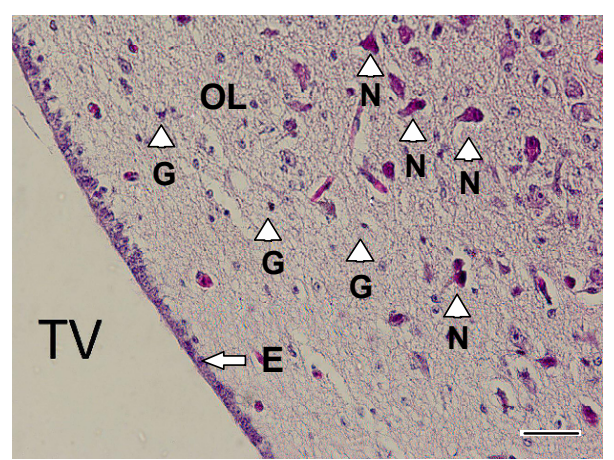

Fig. 10. Tectal ventricle (TV) was lined by simple cuboidal ciliate ependymal cells $(\mathrm{E})$ on the $40^{\text {th }}$ embryonic day. OL: optic lobe, $\mathrm{N}$ : neuron, G: neuroglia $(\mathrm{H} \& \mathrm{E}$, bar $=10 \mu \mathrm{m})$.

\section{Special staining results}

Luxol Fast Blue/Cresyl Echt Violet staining demonstrated that Nissl bodies and tiny myelin fibres were found from the $30^{\text {th }}$ embryonic day onward and that their concentrations (especially in case of myelin fibres) increased with age (Figs 11 \& 12). Malory PTAH staining demonstrated existence of glial fibres (neurophils) from the $36^{\text {th }}$ embryonic day (Figs $13 \& 14$ ). 


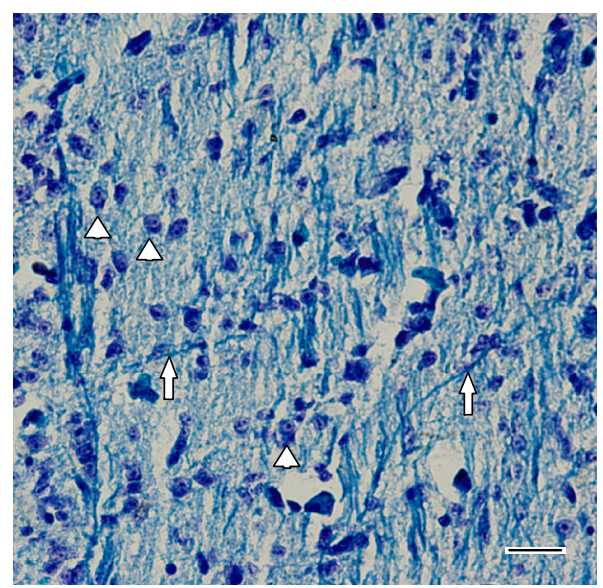

Fig. 11. Luxol Fast Blue/Cresyl Echt Violet staining of optic lobe section, $30^{\text {th }}$ embryonic day. Arrow: myelin fibres, arrowhead: neuron containing Nissl body (bar $=20 \mu \mathrm{m})$.

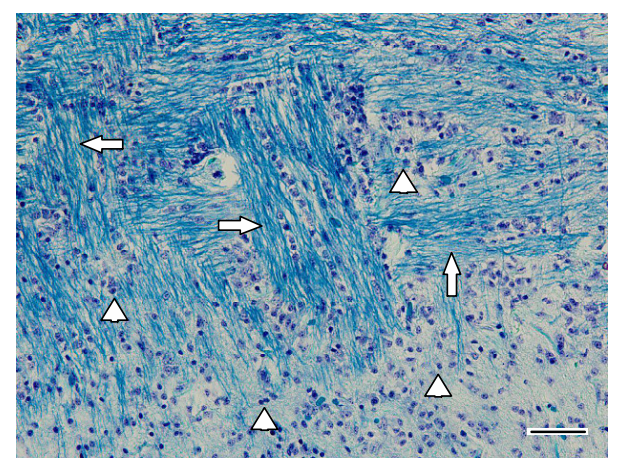

Fig. 12. Luxol Fast Blue/Cresyl Echt Violet staning of the optic lobe section (white matter), $40^{\text {th }}$ embryonic day. Arrow: myelin fibres, arrowhead: neuroglia $($ bar $=25 \mu \mathrm{m})$.

\section{DISCUSSION}

In birds, the central nervous system functions are similar to those of mammals. Its role is to integrate sensory impulses from the environment, to store learned information and coordinate voluntary and involuntary functions and movements. Avian brain research started in the early $20^{\text {th }}$ century (Baumel, 1993). The large optic tectum in avian species can be attributed to the fact that the birds have a very well developed sense of vision (Martin et al., 2007; Douglas et al., 2009; HerculanoHouzel, 2011; Kawabe et al., 2013).

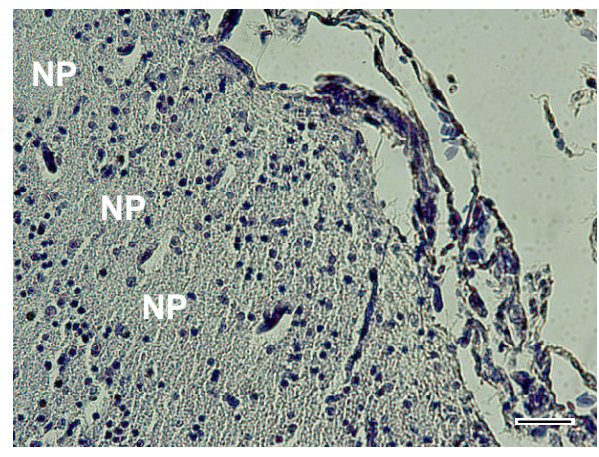

Fig. 13. Mallory PTAH staning of optic lobe section, $30^{\text {th }}$ embryonic day. NP: neuropil (bar $=20 \mu \mathrm{m}$ ).

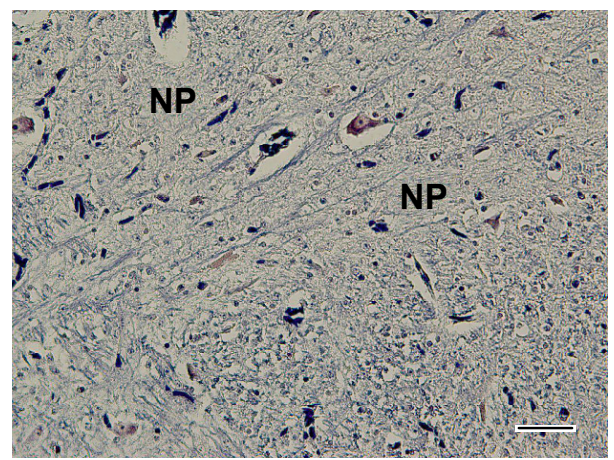

Fig. 14. Mallory PTAH staning of optic lobe, $36^{\text {th }}$ embryonic day. NP: neuropil (bar $=25 \mu \mathrm{m}$ ).

This midbrain tectum opticum more closely resembles the cerebral cortex of mammals than any part of the avian forebrain because in birds there is no neocortex, although superficial corticoid areas are found in the cerebrum. The avian optic lobe is remarkable for its size and for the 
laminar arrangement of the nerve cells (Douglas et al., 2009). In ostrich embryo, the optic lobes are structures that appear on the dorsolateral and medial aspect of the brain. These observations were in accordance with reports in both white crested Polish chickens and uncrested chicken breeds (Frahm et al., 1998).The dorsal surface of the optic lobes was convex. This finding was consistent with data for Sturnus vulgaris (Dhage et al., 2013), in Vencobb broilers (Kumar et al., 2016) and in African ostriches (Karakoura et al., 2015). The entire optic lobes were not visible on the dorsal view. The optic lobes were partially hidden under cerebral hemispheres as in Vencobb broilers (Kumar et al., 2016). However, these optic lobes (tecta) were very large and prominent rounded or spherical bodies of the midbrain on the lateral view. This finding was similar to observations in pigeons (Husband \& Shimizu, 2001). The avian optic lobes' gray matter consisted of neurons, their dendrites, glial cells, blood vessels, and small amount of nerve fibers that may be myelinated (Batah et al., 2012) as found in our study as well. The commonest appearance of the optic lobe cortex comprised small to medium-sized neurons supported by neuroglia as also found out by Karkoura et al. (2015). Neurons were characterised by multiform cells (rounded, pyramidal and occasionally multipolar). Thus, these findings were in contrast with findings of Kumar et al. (2016) in Vencobb broilers. Neurons were characterised by pale, large and acentric nuclei containing obvious nucleolus and Nissl granules in the cytoplasm as already reported (Yokyma et al., 2004; Anthony et al., 2010). Our findings were in line with those of Reiner (2005) in pigeons confirming that the white matter composed of dense bundles of nerve fibres and glial cells. The outer optic lobe layer was molecular layer and its cortex included alternative layers of neural cells and fibres.

Previous studies showed that the tectum in birds was a laminated structure consisting of 15 layers (Douglas et al., 2009). This study demonstrated that the optic lobe cortex showed a special arrangement for neurons: small to mediumsized spherical neurons were arranged in three layers whose thickness increased toward deeper layers. Larger neurons (rounded and pyramidal) were present internally. Occasionally, few multipolar neurons were available in this part acting as interneurons. The large-sized nucleus was visible in the base of optic lobe even on the $30^{\text {th }}$ embryonic day. The tectal nuclei (lateral mesencephalic nuclei) were composed of few multipolar neurons supported by neuroglia. This finding agreed with previous findings about other brain nuclei in the African ostrich (Karakoura et al., 2015). The ratio of tectal ventricles volume to the cortical thickness of optic lobe decreased from $30^{\text {th }}$ to $40^{\text {th }}$ embryonic day. The tectal ventricle was lined with pseudostratified columnar ciliated ependymal cells. As embryonic age increased, the optic lobe thickness increased too. This finding, in line with those of Martin et al. (2007) in Kiwi, confirmed that with the increase of visual ability, tectal thickness increased as well. The aim of special staining (Luxol Fast Blue/Cresyl Echt Violet) demonstrated presence of Nissl bodies and myelin fibres in optic lobe on $30^{\text {th }}, 36^{\text {th }}$ and $40^{\text {th }}$ embryonic days and those concentrations increased with age increase. The PTAH staining revealed existence of glial fibres in optic lobe on $30^{\text {th }}, 36^{\text {th }}$ and $40^{\text {th }}$ embryonic day. No similar findings were found to compare with these histochemical results. 
The morphological stdudies on the embryonic brain are very important for comparative and developmental studies on the brain as no research on ostrich brain morphology during embryonic period is available.

\section{ACKNOWLEDGEMENTS}

This article was a part from the thesis prepared by Zahra Koushafar to fulfill the requirements for earning the $\mathrm{PhD}$ degree in comparative histology. The authors gratefully acknowledge the financial support for this work (Project no: 23222) provided by the Ferdowsi University of Mashhad (FUM).

\section{REFERENCES}

Al-Nasser, A., K. Al-Khalaifa, K. Holleman \& W. Al-Ghalaf, 2003. Ostrich production in the arid environment of Kuwait. Journal of Arid Environments, 54, 219-243.

Batah, A. L., M. S. Ghaje \& N. S. Aziz, 2012. Anatomical and histological study for the brain of the locally breed chicken (Gallus domesticus). Journal of Thi-qar Science, 3, 47-53.

Bancroft, J. D., A. Stevens \& D. R. Turner, 1996. Theory and Practice of Histological Techniques, $4^{\text {th }}$ edn, Churchill Livingstone, New York.

Bejaei, M. \& K. M. Cheng, 2014. A survey of current ostrich handling and transport practices in North America with reference to ostrich welfare and transportation guidelines set up in other countries. Poultry Sciences, 93 , 296-306.

Dhage, S. A., N. A. Shehan, S. A. Ali \& F. H. Aziz , 2013.Anatomical and histological study of cerebral in Sturnus vulgaris. Basrah Journal of Veterinary Reserch, 12, 221-227.

Douglas, R., W. Wylie, G. Cristian \& M. P. Janelle, 2009. The optic tectum of birds: Mapping our way to understanding visual processing. Canadian Journal of Experimental Psychology, 63, 328-338.

Hall, M. I., 2008.The anatomical relationships between the avian eye, orbit and sclerotic ring: implications for inferring activity patterns in extinct birds. Journal of Anatomy, 212, 781-794.

Herculano-Houzel, S., 2011. Brains matter, bodies may be not: The case for examining neuron numbers irrespective of body size. Annals of the New York Academy of Sciences, 12, 191-199.

Husband, S. \& T. Shimizu, 2001. Evolution of the avian visual system. In: Avian Visual Cognition, ed R. G. Cook, https://pigeon.psy.tufts.edu/avc/husband/d efault.htm (8 November 2017 date last accessed).

Frahm, H. D. \& G. Rehkamper, 1998. Allometric comparison of the brain and brain structures in the white crested Polish chicken with uncrested domestic chicken. Brain Behaviour Evolution, 52, 292-307.

Baumel, J. J., 1993. Nomina Anatomica Avium, $12^{\text {th }}$ edn, pp. 516-520.

Karakoura, A. A., M. Alsafy, S. Elgendy \& F. A. Eldefrawy, 2015. Morphological investigation of the brain of the African ostrich (Strutio camelus). International Journal of Morphology, 33, 1468-1475.

Kawabe, S., T. Shimokawa, H. Miki, S. Matsuda \& H. Endo, 2013. Variation in avian brain shape: Relationship with size and orbital shape. Journal of Anatomy, 223, 495-508.

Khanbabaie, R., A. S. Mahani \& R. Wessel, 2007. Contextual interaction of GABAergic circuitry with dynamic synapses. Journal of Neurophysiology, 97, 2802-2811.

Kumar, G. S., B. Kumar, C. R. Pradhan, M. A. Kumar, S. Kumar, D. Behera \& S. K. Parakash, 2016. Studies of the macroscopic and microscopic morphology (hippocampus) of brain in Vencobb broiler. Veterinary world, 9, 507-511.

Letelier, J. C., J. Mpodozis, G. Marin, D. Morales, C. Rozas \& C. Madrid, 2000. 
Spatiotemporal profile of synaptic activation produced by the electrical and visual stimulation of retinal inputs to the optic tectum: A current source density analysis in the pigeon (Columba livia). European Journal of Neuroscience, 12, 47-57.

Luksch, H., 2003. Cytoarchitecture of the avian optic tectum: Neuronal substrate for cellular computation. Reviews in the Neurosciences, 14, 85-106.

Martin, G. R, K. J. Wilson, J. M. Wild, S. Parsons, M. F. Kubke \& J. Corfield, 2007. Kiwi forego vision in the guidance of their nocturnal activities. PLOS ONE, 2,198.

Manns, M., N. Freund, N. Patzke \& O. Gunturkun, 2007. Organization of telencephalotectal projections in pigeons: Impact for lateralized topdown control. Neuroscience, 144, 645-653.

Mescher, A. L., 2010. Junqueira's Basic Histology: Text and Atlas. $12^{\text {nd }}$ edn, McGrawHill Medical, pp. 183-215.

Metzger, M., L. R. Britto \& C. A. Toledo, 2006. Monoaminergic markers in the optic tectum of the domestic chick. Neuroscience, 141, 1747-1760.

Peng , K.,Y. Feng, G. Zhang, H. Liu \& H. Song, 2010.Anatomical study of the brain of the African ostrich. Turkish Journal of Veterinary and Animal Sciences, 34, 235241.

Reiner, A., 2005. A new avian brain nomenclature: Why, how and what. Brain Research Bulletin, 66, 317-331.

Sebesteny, T., D. C. Davies, N. Zayats, A. Nemeth \& T. Tombol, 2002. The ramification and connections of retinal fibres in layer 7 of the domestic chick optic tectum: A Golgi impregnation, anterograde tracer and GABA-immunogold study. Journal of Anatomy, 200, 169-183.

Wang, Y., H. Luksch, N. C. Brecha \& H. J. Karten, 2006. Columnar projections from the cholinergic nucleus isthmi to the optic tectum in chicks (Gallus gallus): A possible substrate for synchronizing tectal channels. Journal of Comparative Neurology, 494, 7-35.

Wenqin, C., L. Huazhen \& L. Guanzhong, 2005. Study on the cytoarchitecture of 5 nuclei of Medulla oblongata in African ostrich (Struthio camelus). Huazhong Nongye Daxue Xuebao, 24, 185-188.

Yokoyama, A., L. Yang, S. Itoh, K. Mori \& J. Tanaka, 2004. Microglia, a potential source of neurons, astrocytes, and oligodendrocytes. Glia, 45, 96-104.

Paper received 22.05.2017; accepted for publication 21.09.2017

\section{Correspondence:}

Dr. Ahmad Ali Mohammadpour Department of Basic Sciences, Faculty of Veterinary Medicine, Ferdowsi University of Mashhad, P.O. Box 1793, Mashhad, Iran, e-mail: mohammadpoor@ferdowsi.um.ac.ir 